\title{
Water Soluble Trianiline Containing Polyurethane (TAPU) as an Efficient Corrosion Inhibitor for Mild Steel
}

\author{
Feng Yang ${ }^{1}$, Xiangyu Li $^{1,2}$, Shihui Qiu ${ }^{2}$, Wenru Zheng ${ }^{2}$, Haichao Zhao ${ }^{2, *}$, Liping Wang ${ }^{2, *}$ \\ ${ }^{1}$ School of Materials Science and Engineering, Shenyang University of Chemical Technology, Shen \\ Yang, 110142, China \\ ${ }^{2}$ Key Laboratory of Marine Materials and Related Technologies, Zhejiang Key Laboratory of Marine \\ Materials and Protective Technologies, Ningbo Institute of Materials Technology and Engineering, \\ Chinese Academy of Sciences, Ningbo 315201, P. R. China. \\ *E-mail: zhaohaichao@nimte.ac.cn, wangliping@nimte.ac.cn
}

doi: $10.20964 / 2017.06 .53$

Received: 2 March 2017 / Accepted: 23 April 2017 / Published: 12 May 2017

\begin{abstract}
Novel trianiline containing water soluble polyurethane (TAPU) was synthesized by polymerization of polyethylene glycol, toluene diisocyanate and amine-capped aniline trimer. The influence of TAPU on the corrosion inhibition of mild steel in $1 \mathrm{M} \mathrm{HCl}$ was investigated using weight loss measurements, potentiodynamic polarization, electrochemical impedance spectroscopy (EIS). The performance of TAPU on the corrosion inhibition of mild steel was also evidenced by scanning electron microscopy (SEM), energy dispersive spectroscopy (EDS) and atomic force microscope (AFM). Results confirmed that the adsorption of TAPU on the mild steel surface and consequent inhibition of the corrosion process. The inhibition efficiency (IE\%) increased with a rise in TAPU concentration, reaching a value up to $97 \%$ at a concentration of $200 \mathrm{mg} / \mathrm{l}$. Potentiodynamic polarization curves showed that the TAPU affected both cathodic and anodic protection and was a mixed type inhibitor in $\mathrm{HCl}$ corrosive medium. Adsorption isotherm studies confirmed that the adsorption of TAPU on the mild steel surface in $1 \mathrm{M}$ $\mathrm{HCl}$ solution obeyed Langmuir adsorption isotherm. EDS analysis determined the adsorption of TAPU molecules on the steel surface, and the absorbed TAPU provided an effective corrosion inhibition in 1 $\mathrm{M} \mathrm{HCl}$ solution from observation of surface morphology by SEM and AFM.
\end{abstract}

Keywords: polyurethane; trianiline; acidic aqueous solution; corrosive inhibitor; mild steel

\section{FULL TEXT}

(C) 2017 The Authors. Published by ESG (www.electrochemsci.org). This article is an open access article distributed under the terms and conditions of the Creative Commons Attribution license (http://creativecommons.org/licenses/by/4.0/). 